\title{
Effect of Heart Failure-induced Electrical Remodeling on the Initiation of Atrial Arrhythmias
}

\author{
Na Zhao ${ }^{1}$, Qince $\mathrm{Li}^{1}$, Kuanquan Wang ${ }^{1}$, Yong Xia ${ }^{1}$, Runnan $\mathrm{He}^{1}$, Xiangyun $\mathrm{Bai}^{1}$, Henggui Zhang ${ }^{1,2}$ \\ ${ }^{1}$ School of Computer Science and Technology, Harbin Institute of Technology, Harbin, China \\ ${ }^{2}$ School of Physics and Astronomy, the University of Manchester, Manchester, UK
}

\begin{abstract}
Heart failure (HF) is a particularly prevalent clinical condition promoting atrial arrhythmias. However, the underlying mechanism is rarely studied. In this study, using a GPU-based simulation, a biophysically detailed computational model of the three-dimensional (3D) sheep atria was implemented to investigate the mechanism by which HF-induced electrical remodeling promoting atrial arrhythmia. At both the single cell and the $3 D$ levels of the sheep atrial model, effects of such HF-induced electrical remodeling on the electrical properties were evaluated. At the cellular level, simulation results demonstrated that the action potential duration (APD) and the amplitude of systolic $\mathrm{Ca}^{2+}$ transient were decreased in all cell types except the PV cell in the HF condition. At the $3 D$ whole organ level, simulation results showed that though localized APDs were shortened, the spatial electrical heterogeneity was maintained in the HF condition, resulting in an increased vulnerability of the tissue for the initiation of the conduction block in response to a premature stimulus. This study provided new insights into understanding the mechanism by which HF promoted atrial arrhythmias.
\end{abstract}

\section{Introduction}

Heart failure (HF) is a particularly prevalent clinical condition, and it is estimated that there are 26 million people worldwide suffering from HF [1]. Many patients with HF may be accompanied by atrial arrhythmias, such as atrial fibrillation (AF). Though it is believed that there is a relationship between atrial arrhythmias and HF [2], the causative relationship between the two is incompletely understood.

Several studies have shown structural and electrical remodeling of the atria as a result of HF. Atrial myocytes from sheep or canine hearts with HF induced by ventricular tachypacing showed reductions of the density of L-type $\mathrm{Ca}^{2+}$ current $\left(I_{\mathrm{CaL}}\right)$, transient outward $\mathrm{K}^{+}$current $\left(I_{\mathrm{to}}\right)$ and slow delayed rectifier current $\left(I_{\mathrm{Ks}}\right)$, and an increase of transient inward $\mathrm{Na}^{+} / \mathrm{Ca}^{2+}$ exchanger $\left(I_{\mathrm{NCX}}\right)$ current compared with normal hearts [3-6]. In addition, $\mathrm{HF}$ has impact on atrial myocyte cellular $\mathrm{Ca}^{2+}$ handling. Yeh et al. used a canine model of dilated cardiomyopathy produced by ventricular tachypacing and found a larger $\mathrm{Ca}^{2+}$ transient, increased sarcoplasmic reticulum (SR) $\mathrm{Ca}^{2+}$ load (caffeine-induced $\left[\mathrm{Ca}^{2+}\right]_{\mathrm{i}}$ release) and prolonged action potential duration (APD) in HF myocytes [7]. On the contrary, Clarke et al. showed a reduction in $\mathrm{Ca}^{2+}$ transient, shortened APD and reduced $\mathrm{Ca}^{2+}$ transient with an ovine model of HF [3]. All of these changes may contribute to abnormalities in the propagation of electrical excitation waves. However, the effect of electrical remodeling at the cellular level induced by HF on atrial electrical properties at the tissue level that promotes atrial arrhythmias has not yet been fully elucidated.

In this study, the effects of HF-induced changes of several ionic currents ( $\left.I_{\mathrm{CaL}}, I_{\mathrm{to}}, I_{\mathrm{Ks}}, I_{\mathrm{NCX}}\right)$ were based on experimental data of sheep or canine atria cells, which were incorporated into the sheep atrial cell model developed by Butters et al. [8]. At the single cell level, effects of such HF-induced electrical remodeling on the action potential (AP) profile and APD as well as the intracellular $\mathrm{Ca}^{2+}$ transient were evaluated. Furthermore, the single cell models were incorporated into an anatomically accurate and biophysically detailed 3D model of the sheep atria. We used a GPU-based simulation [9] effectively to quantify the effects of the HF-induced remodeling on: (1) APD dispersion across the atria; and (2) vulnerability of the tissue to the initiation of conduction block in response to a premature stimulus that underlies the genesis of atrial arrhythmias.

\section{Methods}

\subsection{Atrial cell models with HF-induced electrical remodeling}

We used a set of cellular models of sheep atrial myocytes developed by Butters et al. [8] that took into considerations of the intrinsic electrophysiological 
Table 1. Model parameters of HF-induced remodeling.

\begin{tabular}{llll}
\hline Process & HF-1 & HF-2 & HF-3 \\
\hline$I_{\mathrm{CaL}}$ & $-36 \% \tau_{\text {inac }}+75 \%$ & $-50 \%$ & $-30 \%$ \\
$I_{\text {to }}$ & No change & $-65 \%$ & $-50 \%$ \\
$I_{\mathrm{Ks}}$ & No change & $-40 \%$ & $-30 \%$ \\
$I_{\mathrm{NCX}}$ & No change & $+110 \%$ & $+45 \%$ \\
\hline
\end{tabular}

heterogeneity in distinctive regions of the atria, including pectinate muscles (PM), right atrium (RA), crista teminalis (CT), Bachmann's bundle (BB), left atrium (LA) and pulmonary vein (PV). At the cellular level, we calculated the $\mathrm{APD}_{90}$ (APD values at $90 \%$ ) and $\mathrm{Ca}^{2+}$ transient amplitude of LA myocyte at $0.5 \mathrm{~Hz}$ for the change of $I_{\mathrm{CaL}}$ (reduction of $36 \%$ and increasing inactivation time constant of the channel $\left(\tau_{\text {inac }}\right)$ by $75 \%$, as shown in the case of HF-1 in Table 1) under HF based on the experiment data of the sheep [3]. As data from sheep may be incomplete, we also assumed similar changes of $I_{\mathrm{CaL}}, I_{\mathrm{to}}, I_{\mathrm{Ks}}$ and $I_{\mathrm{NCX}}$ as shown in canine HF atrial cells [4,5] as shown in cases of HF-2 and HF-3 in Table 1. We compared the obtained results in the three HF conditions with those in normal condition for the calculated APD and $\mathrm{Ca}^{2+}$ transient amplitude, which were validated by experimental data. After validation, we evaluated the effect of HF-induced electrical remodeling on electrical properties of atrial myocytes.

\subsection{GPU-based 3D sheep atrial model and analysis of APD distribution and tissue vulnerability}

The anatomically accurate and biophysically detailed 3D model of the sheep atria developed by Butters et al. [8] was used in this study. In order to run the simulation of the 3D whole atrial model, we adopted the efficient GPU CUDA algorithm developed by Xia et al. [9].

In the organ model, three consecutive stimuli were applied to the sinoatrial node (SAN) region at a basic cycle length (BCL) of 500 ms to initiate the conduction of electrical excitation waves in the $3 \mathrm{D}$ atria for control and HF conditions. The APD distribution across the whole atria for each condition was mapped by calculating the $\mathrm{APD}_{90}$ of every atrial myocyte.

The vulnerable window (VW) for unidirectional conduction block $\left(\mathrm{VW}_{\mathrm{CB}}\right)$ in response to a premature stimulus was calculated in control and HF conditions. In simulations, standard S1-S2 pacing protocol was applied to the tissue regions of the CT/PM junction, following the same approach as used in Colman et al. [10,11]. Three S1 stimuli were applied at a cycle length of $500 \mathrm{~ms}$ for control and HF models, followed by a shorter-coupled S2 stimulus. The range of the time interval between the last S1 and S2 (i.e., the coupling intervals) for which the electrical wave could propagation in one atrial tissue region but was blocked in the other was considered as the $\mathrm{VW}_{\mathrm{CB}}$.

\section{Results \\ 3.1. Effects of HF-induced electrical remodeling at single cell level}

With consideration of remodelled $I_{\mathrm{CaL}}$ alone in sheep LA cell under the HF (HF-1) condition, the simulated AP at $0.5 \mathrm{~Hz}$ demonstrated a more triangular profile (Figure $1 A a)$, a decreased magnitude and duration of the $\mathrm{Ca}^{2+}$ transient (Figure $1 \mathrm{Ba}$ ), and a reduced $\mathrm{APD}_{90}$ by $9.5 \%$ (Figure $1 A b$ ), and a reduced amplitude of systolic $\mathrm{Ca}^{2+}$ transient by $13.02 \%$ (Figure $1 B b$ ) as compared to the control condition. These simulation results were much smaller compared to the experimental observations by Clarke et al. [3], who showed a reduction in $\mathrm{APD}_{90}$ by $22 \%$ and a reduction in the amplitude of systolic $\mathrm{Ca}^{2+}$ transient by $46 \pm 17 \%$. When cases of HF- 2 and HF-3 were considered, the simulated results showed a reduction in effective refractory period (ERP) and $\mathrm{APD}_{90}$, which were opposite to the observation of Cha et al. [4] and $\mathrm{Li}$ et al. [5], who showed increases in both of them. Therefore, in the following studies, HF-1 condition was used.

Figure 2 represents the effect of HF-induced electrical remodeling on the regional heterogeneity in atrial electrical action potentials. In this figure, AP traces from cells in two nearby regions of the CT and PM (Figure 2a) and their corresponding $\mathrm{APD}_{90}$ (Figure $2 b$ ) were shown for the control and HF conditions. It was shown that the HF-induced ion channel remodelling resulted in reduction of APD ${ }_{90}$ in all cell types except for the PV cell.
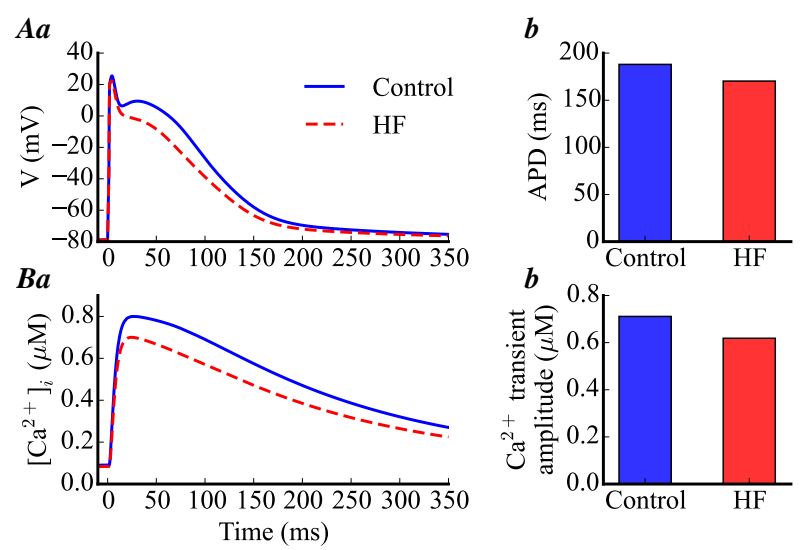

Figure 1. Effects of HF-induced remodeling on LA cell AP, APD $90,\left[\mathrm{Ca}^{2+}\right]_{\mathrm{i}}$ and their amplitude. A, APs $(a)$ and their APD in control (blue) and HF-induced remodeling (red) conditions. $\mathrm{B}, \mathrm{Ca}^{2+}$ transient (a) and $\mathrm{Ca}^{2+}$ transient amplitude (b) in control (blue) and HF-induced remodeling (red) conditions. 

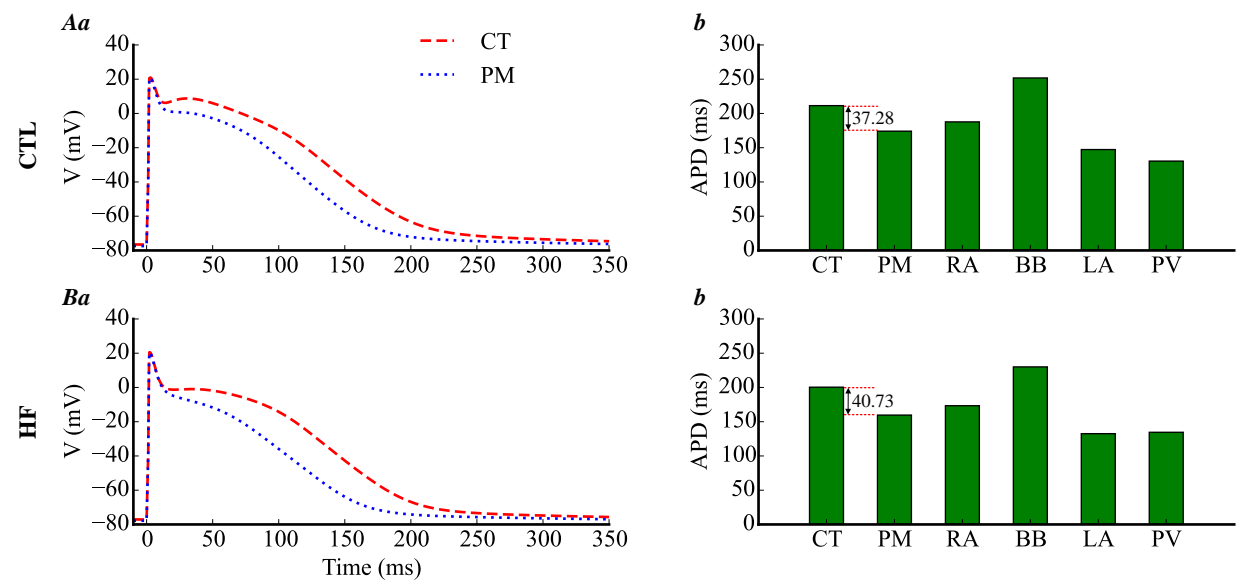

Figure 2. Effect of HF-induced remodeling on AP regional heterogeneity. Comparisons of AP were made between the CT and PM in control $(A a)$ and HF-induced remodeling $(B a)$ conditions, and $\mathrm{APD}_{90}$ for atrial regional cells in control $(A b)$ and HF-induced remodeling $(B b)$ conditions.

However, the electrical heterogeneity at the junction region of CT/PM junction sustained, but became negligible at the $\mathrm{LA} / \mathrm{PV}$ junction. The $\mathrm{APD}_{90}$ difference between CT and PM was 37.28 ms in control versus $40.73 \mathrm{~ms}$ in HF, and for LA and PV it became $16.87 \mathrm{~ms}$ in control versus $2.01 \mathrm{~ms}$ in HF. Therefore, the regional heterogeneity still retained in most regions even though the HF-induced remodeling resulted in a shortened APD in most atrial cell type. This was similar to the observation of Colman et al. [10], who showed that AFinduced electrical remodeling shortened APD, but sustained spatial heterogeneity at CT/PM junction.

\subsection{Effects of HF-induced electrical remodeling at $3 D$ whole organ level}

Figure 3 shows the geometrical structure in vertical and bottom views (Figure 3A) and spatial distribution of $\mathrm{APD}_{90}$ (Figure $3 \mathrm{~B}$ ) for the tissue around the CT and PM regions. In HF condition, the measured APD of every myocyte across the CT and PM regions was slight shorter (ranging from $1 \mathrm{~ms}$ to $17 \mathrm{~ms}$ ) than the one in control condition. The $\mathrm{APD}_{90}$ of $\mathrm{PM}$ at the triangular region (red region in Figure 3B) was longer than the one of CT. This might be attributed to the cell-to-cell electrical coupling at tissue level between $\mathrm{PM}$ and $\mathrm{BB}$, as $\mathrm{PM}$ at the triangular location was close to the $\mathrm{BB}$ region at which isolated cells had the longest $\mathrm{APD}_{90}$ in the whole atrium. Although the $\mathrm{APD}_{90}$ in CT and PM regions at the tissue level was also reduced, but the $\mathrm{APD}_{90}$ heterogeneity at the junction was preserved. For example, the difference in $\mathrm{APD}_{90}$ between single location within $\mathrm{CT}$ and $\mathrm{PM}$ regions respectively close to $\mathrm{CT} / \mathrm{PM}$ junction was $7.35 \mathrm{~ms}$ in control versus 10.07 in HF (Figure 4A).

Figure 4 represents the difference in $\mathrm{APD}_{90}$ and the $\mathrm{VW}_{\mathrm{CB}}$ at the region of CT/PM junction in 3D sheep atria in control and HF-induced electrical remodeling models.

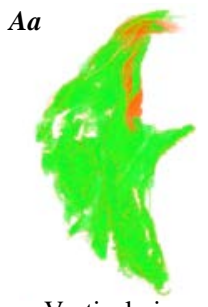

Vertical view

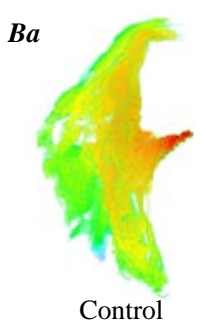

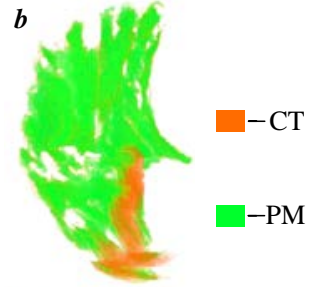

Bottom view

b

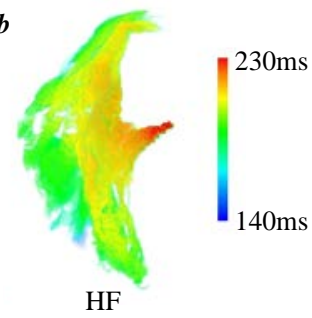

Figure 3. Geometry of the CT (orange) and PM (green) in sheep right atrium in the vertical $(A a)$ and bottom $(A b)$ views, and $\mathrm{APD}_{90}$ spatial distribution maps for control $(B a)$ and $\mathrm{HF}(B b)$ models.
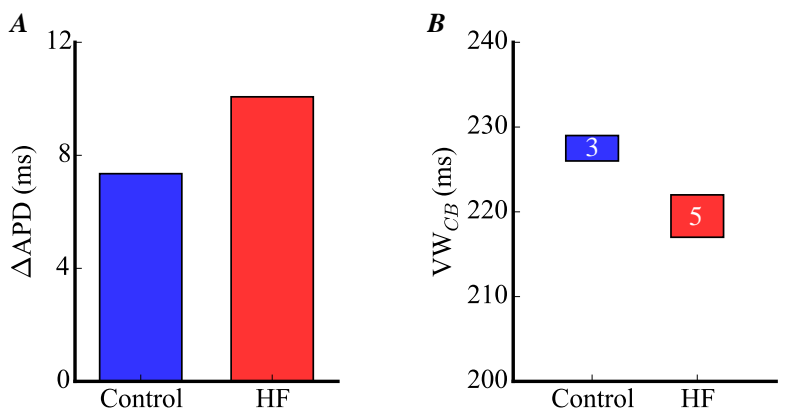

Figure 4. Difference in $\mathrm{APD}_{90}(A)$ and the $\mathrm{VW}_{\mathrm{CB}}(B)$ at the region of CT/PM junction in the 3D sheep atria in control and HF-induced electrical remodeling models. 

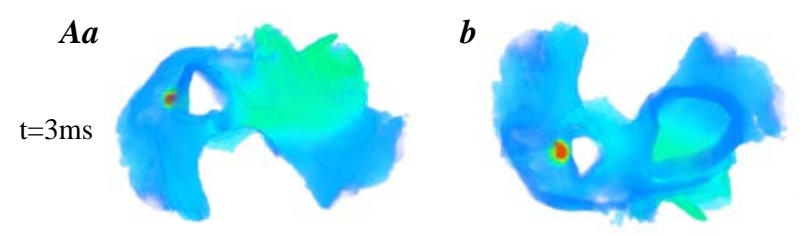

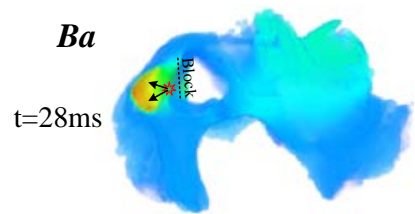

Vertical view

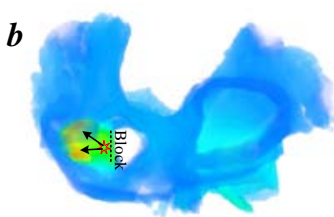

Bottom view

$$
-85 \mathrm{mV} \quad 10 \mathrm{mV}
$$

Figure 5. Snapshots of conduction block at the CT/PM junction after a premature S2 stimulus: $\mathrm{t}=3 \mathrm{~ms}(A), 28 \mathrm{~ms}$ $(B)$ in vertical $(a)$ and bottom $(b)$ views.

HF resulted larger $\mathrm{VW}_{\mathrm{CB}}$ with shorter coupling intervals compared with control (Figure 4B), which were $3 \mathrm{~ms}$ (from $226 \mathrm{~ms}$ to $228 \mathrm{~ms}$ ) in control and $5 \mathrm{~ms}$ (from 217 $\mathrm{ms}$ to $221 \mathrm{~ms}$ ). Figure 5 shows the conduction block at the CT/PM junction after a premature S2 stimulus. The conduction was permitted in the PM, but failed in the CT (Figure 5B). This might be attributed to the incomplete recovery of CT tissue with longer $\mathrm{APD}_{90}$ than $\mathrm{PM}$ tissue from the previous S1 stimulus, underlying the genesis of atrial arrhythmias.

\section{Discussion and conclusion}

In this study, we investigated the effect of HF-induced electrical remodeling on the initiation of atrial arrhythmias by evaluating the changes of electrical properties at both single cell level and 3D atria level. It was shown that at the single cell level, due to reduced $I_{\mathrm{CaL}}$, $\mathrm{HF}$ resulted in a reduced $\mathrm{APD}_{90}$ and $\mathrm{Ca}^{2+}$ transient amplitude for all cell types except for the PV cell. However, at the tissue level, the electrical heterogeneity was preserved in the junction between CT and PM, though reduced at the LA and PV junction. Especially the difference of $\mathrm{APD}_{90}$ between $\mathrm{CT}$ and $\mathrm{PM}$ regions was augmented in HF condition as compared to control condition, resulted in an increased tissue's vulnerability for initiation of blocked excitation waves by a premature stimulus at the 3D atria level. Our study provided more insights into understanding the mechanism by which $\mathrm{HF}$ promotes atrial arrhythmias.

\section{Acknowledgements}

This work is supported by the National Natural Science Foundation of China (NSFC) under Grant No.
61571165, No. 61572152.

\section{References}

[1] Ambrosy AP, Fonarow GC, Butler J, Chioncel O, Greene SJ, Vaduganathan M, Nodari S, Lam CSP, Sato N, Shah AN, Gheorghiade $M$. The global health and economic burden of hospitalizations for heart failure. J Am Coll Cardiol 2014;63:1123-1133.

[2] Andrade J, Khairy P, Dobrev D, Nattel S. The clinical profile and pathophysiology of atrial fibrillation: Relationships among clinical features, epidemiology, and mechanisms. Circ Res 2014;114:1453-1468.

[3] Clarke JD, Caldwell JL, Horn MA, Bode EF, Richards MA, Hall MCS, Graham HK, Briston SJ, Greensmith DJ, Eisner DA, Dibb KM, Trafford AW. Perturbed atrial calcium handling in an ovine model of heart failure: Potential roles for reductions in the L-type calcium current. Journal of Molecular and Cellular Cardiology 2015;79:169-179.

[4] Cha T, Ehrlich JR, Zhang L, Shi Y, Tardif J, Leung TK, Nattel S. Dissociation between ionic remodeling and ability to sustain atrial fibrillation during recovery from experimental congestive heart failure. Circulation 2004; 109:412-418

[5] Li D, Melnyk P, Feng J, Wang Z, Petrecca K, Shrier A, Nattel S. Effects of experimental heart failure on atrial cellular and ionic electrophysiology. Circulation 2000; 101: 2631-2638.

[6] Cha T, Ehrlich JR, Zhang L, Nattel S. Atrial ionic remodeling induced by atrial tachycardia in the presence of congestive heart failure. Circulation 2004;110:1520-1526.

[7] Yeh YH, Wakili R, Qi XY, Chartier D, Boknik P, Kääb S, Ravens U, Coutu P, Dobrec D, Nattel S. Calcium-handling abnormalities underlying atrial arrhythmogenesis and contractile dysfunction in dogs with congestive heart failure. Circ Arrhythmia Electrophysiol 2008;1:93-102.

[8] Butters TD, Aslanidi OV, Zhao J, Smaill B, Zhang H. A novel computational sheep atria model for the study of atrial fibrillation. Interface focus 2013;3:20120067.

[9] Xia Y, Wang K, Zhang H. Parallel optimization of 3D cardiac electrophysiological model using GPU. Computational and mathematical methods in medicine 2015;2015.

[10] Colman MA, Aslanidi OV, Kharche S, Boyett MR, Garratt C, Hancox JC, Zhang H. Pro-arrhythmogenic effects of atrial fibrillation-induced electrical remodelling: insights from the three-dimentional virtual human atria. J physiol 2013;591:4249-4272.

[11] Colman MA, Varela M, Hancox JC, Zhang H, Aslanidi OV. Evolution and pharmacological modulation of the arrhythmogenic wave dynamics in canine pulmonary vein model. Europace 2014;16: 416-423.

Address for correspondence.

Henggui Zhang

Room 3.07, Shuster building

Manchester, M13 9PL, UK

henggui.zhang@manchester.ac.uk 